Pure \& Appl. Chem., Vo1. 49, pp. 827 - 837. Pergamon Press, 1977. Printed in Great Britain.

\title{
25 YEARS OF LIGAND-FIELD-THEORY
}

\section{Hermann Hartmann}

Akademie der Wissenschaften und der Literatur zu Mainz Geschwister Scholl-Str. 2, 65 Mainz

\begin{abstract}
This paper gives a short description of the historical development of ligand field theory, including a presentation of the basic ideas and of the most important results, together with personal remembrances out of the pioneering period.
\end{abstract}

In 1951 I published under my name and the name of my friend and coworker F.E. Ilse (Ref. 1) our first papers concerning a theory of the term systems of complex ions developed on the basis of the electrostatic theory of complex compounds which had been founded by A. Magnus (Ref. 2) in 1922. F.E. Ilse was my second doctorand. His thesis (Ref. 3) was finished in 1946. He died in 1949 .

In the same year 1951 at the international spectroscopists' meeting held in Basel, Switzerland, I gave a report about what we meanwhile called "Iigandenfeldtheorie". I used that word in my lecture held in Basel and I think that was the first time it was used, because all papers concerning the theory of term systems of complex ions on the basis of the electrostatic theory written by other scientists appeared at least one year later.

In my talk in Basel I also mentioned the experimental results which my coworker H.L. Schläfer (Ref. 4) had described in his thesis in 1950. Schläfer had investigated the light absorption of $\mathrm{Ti}(3+)$-complex ions as the most simple cases suited to test the propositions of our ligand-field-theory.

My friends in the organizing committee of this congress 25 vears after our first publications have entrusted me with the task to speak about 25 years of ligand-field-theory.

For me it is a bit difficult to do that, because I am quite sure that I am not the right man to take you all on a hiọhly intensive tour d'horizon through a quarter of a century of theoretical complex chemistry. I resolved to do the following: at first I shall try to describe the situation in theoretical complex chemistry in 1945. Then I shall explain the basic idea of our ligand-field-theory. I think that will be useful because modern complex chemists work with much more advanced theoretical programs and perhaps are not so well aware about the first steps. In that part of my talk I shall say how and why ligand-field-theory works.

Then I shall pick out two typical examples and show which types of results in my opinion are the most important ones. Then I want to say only a few words about further developments which led to the present satisfying situation in theoretical complex chemistry, but point out the relation of ligand-field-theory to a kind of complementarity principle between knowledge and understanding. After that I shall collect some personal facts, which may be useful for those who are interested in the phenomena of the genesis of science. All other people in the audience I beg to excuse this short introductory chapter of my talk. At the end I want to say something about my personal relation to ligand-field-theory.

\section{1 .}

Alfred Werner (Ref. 5) in his Nobel-Lecture from 1914 has been very cautious with statements about the nature and the action of chemical forces in comolex compounds. In his famous book from 1905 he had treated complex chemistry from a purely phenomenological point of view. 
The situation was suddenly changed, when A. Magnus (Ref. 2) in 1922 published his theory of complex compounds on an electrostatic basis, as introduced into chemistry in 1916 by the famous paper of A. Kossel (Ref. 6). You may remember that Kossel in the same paper had introduced the principle of electron-shells. I have here a book published in 1919. Its author, R. Weinland, (Ref. 7) a wellknown complex-chemist was a phenomenologist like Werner. But here is a hand-written dedication by the author to his friend Alfred Magnus. It may be that this copy of Weinland's book marks the essential stimulation for the beginning of a physical theory of complex compounds.

The principal merit of Magnus' theory consisted in a quantitative explanation of the relation between main coordination number and the charge number of the central ion. On the other hand the theory was unable to explain uncommon structural types like the square complexes with coordination number four and the main disadvantage was that there was no place in Magnus' theory for the explanation of abnormal reduced magnetism.

The introduction of the electron pair concept into theoretical chemistry by Lewis (Ref. 8) in 1916 was followed by the postulate of closed shells by Langmuir (Ref. 9) in 1923. Sidgwick (Ref. 10) applied the shell principle to complex entities and extended this idea later.

While in case of compounds of elements of the three first groups of the periodic table the characteristic electron numbers according to Langmuir and Lewis were 2 and 8 , for compounds of transition metals 18 had to be introduced as the characteristic electron number.

It was very important for the further development of theoretical complex chemistry, that the application of the shell principle led to an understanding of abnormal reduced magnetism at least in principle. But there were still discrepancies between theory and experiment. So for example Sidgwick's theory could not explain that for $\mathrm{Ni}(2+)$ and coordination number four different magnetic behaviour was observed for different geometrical structures.

Lewis' and Sidgwick's theoretical concepts were at first considered as well suited tools for the description of covalency. So - and that was the important point - the success of the shell principle in explaining reduced magnetism of transition metal compounds was considered as some kind of proof, that complexes normally (and that means with only few exceptions) are good covalent entities.

So in the latter years of the twenties most chemists were convinced that Magnus' theory, to which they had attributed so much weight at first, was in fact very unimportant because it concerned only the rare "electrostatic" complexes, whilst it was useless for the bulk of normal or "penetration" complexes.

In 1927 Heitler and London (Ref. 11) found the still today accepted and as I think truly final physical explanation of the mysterious covalent bond. So two years after the foundation of quantum mechanics the central problem of theoretical chemistry was solved.

In the period of quantum chemistry, which was founded by Heitler and Iondon, Pauling (Ref. 12) studied the bond problem for covalent or penetrationcomplexes. He investigated the construction of bond eigenfunctions by linear combination or hybridisation of one-center-functions under the condition of a pretended symmetry and the assumption of validity of the principle of maximum overlapping. What he did could in a broader sense be visualized as the use of valence-bond-theory for the special case of covalent complexes.

The generally highly esteemed and really very important results of Pauling were the solution of the problem left over by the Sidgwick-theory, namely the understanding of the relation between magnetic behavior and structure of $\mathrm{Ni}(2+)$-complexes and related other things.

Dauling's success from 1931 consolidated the opinion of the covalent nature of the chemical bond in complexes. Later Pauling pointed out, that probably complexes are not purely covalent entities. Using the language of valencebond-theory the ground state of a complex ion according to his opinion is to be described by a linear combination of covalent and electrovalent valencebond-eigenfunctions with preponderant covalency. 
With regard to the situation in 1945 it is important to recognize that whatever Pauling.did with complexes referred to the ground state. He never looked for excited states, and the reason why he did not is very simple. Complete calculation of for example excitation energies even for the case of pure covalency with the scheme of valence-bond-theory of comolex ions is so extremely complex and uncertain because of many necessary simplifications that at least around 1930 it was hopeless.

Molecular orbital theory besides valence-bond-theory originated rather early as a second qualitative theoretical system. Hund, Mulliken and Herzberg (HMH-theory, Ref. 13) were the founders of this system in the years 1931 to 1932. So it was quite natural, that van vleck (Ref. 14) and Mulliken (Ref. 15) independently in 1933 studied complexes from that point of view.

Van Vleck and Sherman in 1934 wrote an article (Ref. 16) on the Quantum Theory of Valence for Reviews of Modern Physics. In this article there is a table on which central ion portions and ligand portions of molecular orbitals for the complex ions $\left[\mathrm{Fe} \mathrm{F}_{6}\right]^{3-}$ and $\left./ \mathrm{Ni}(\mathrm{CN})_{4}\right]^{2}$ are given explicitly. The table does not contain molecular orbitals occupied in $\pi$-bonding, but this point is discussed in the following section of the article. This is interesting because some people think that MO-theory of complexes was invented after our ligand-field-theory, as an imorovement.

Van Vleck as well as Mulliken in their papers for ordering MO's have used as auxiliary tool the system of irreducible representations of the corresponding symmetry groups. In 1933 that had become a quite common method after wigner (Ref. 17) in 1927 had detected the relation between quantum-mechanics and group-theory, and in 1930 Bethe (Ref. 18) had investigated the application of finite groups.

But what did Van Vleck and Mulliken do with the molecular orbitals they had constructed? At first, all conclusions were drawn for the ground state. There were no calculations of energies, but only arguments with bonding and antibonding properties of the MO's. The reason was again the high complexity of the many-electron system called a complex ion.

The papers on MO-theory of complexes at that time did not change the general opinion of essential covalency in complexes.

After 1933 for many years nearly nothing happened in theoretical complex chemistry. We had a real stagnation until 1945 .

\section{2.}

The basic idea of the ligand-field-theory from the outset in 1945 seemed to be in total contradiction to general opinion about the nature of the chemical bond in complex entities.

This idea contains two parts. For the explanation of the first part let me use the language of valence-bond-theory. Covalent and electrovalent valencebond-structures of complexes may be represented by $\psi_{\text {cov }}$ and $\psi \mathrm{e} / \mathrm{v}$. Electronic states of a complex are represented by a linear combination and we write

$$
c_{\operatorname{cov}} \psi_{\operatorname{cov}}+c_{e / v} \psi_{e / v}
$$

to express symbolically that the linear combination normally contains covalent and electrovalent components.

The first part of the basic idea of ligand-field-theory consists in the simplification

$$
\mathrm{C}_{\mathrm{cov}} \psi_{\mathrm{cov}}+\mathrm{c}_{\mathrm{e} / \mathrm{v}} \psi_{\mathrm{e} / \mathrm{v}} \rightarrow \psi_{\mathrm{e} / \mathrm{v}}
$$

We disregard the covalent components. So we treat the complex entities as if they were purely electrovalent. But the error in the energies which are calculated from the eigenfunctions of type $\psi \mathrm{e} / \mathrm{v}$ is only relatively small because the variation principle of quantum mechanics as described in Schrödingers first paper has the consequence that an error in the state- 
function leads only to a less important error in the energy. That is the physical confirmation for the procedure I. The same argument could justify a purely covalent theory also. But a covalent theory would not allow the second simplifying step.

Until now it was tacitly assumed that the $\psi$ 's are functions of the coordinates of all valence electrons. Such electrons are those of the central ion and the outer electrons of the ligands. That corresnonds to the ideas of Pauling and of Van Vleck and Mulliken.

The second simplifying step now consists in disregarding an explicit inclusion of the outer electrons of the ligands. From now on only valence electrons of the central ion are treated explicitly and the outer electrons of the ligands come into play in so far as they participate in producing the ligand-field, which acts on the valance-electrons of the central ion. If Wion resembles a state function depending on the coordinates of the valence electrons at the central ion only, we can formulate the second part of the basic idea or the second simplifying step as

$$
\psi_{e / v} \rightarrow \psi_{\text {ion }}
$$

To justify this step from the outset has been more difficult. Only a general experience with electrostatic theories in chemistry could overcome critical objections.

The main task of the theory presented itself now in the form of a perturbation problem. The splitting diagram of the involved ion-terms could be determined by group theoretical methods. The approximate eigenfunctions of zeroth order could be taken from Slater's theory of atomic states. The parameters of the effective Hamiltonian as model quantities had to be roughly estimated from distances, charges, dipolemoments, polarizabilities etc.

This situation was related to a similar but not identical situation in the so called crystal-field-theory. In this theory the aim is to get results about the magnetic properties of definitely ionic lattices in their ground state. In contrast to that aim the principal goal of ligand-field-theory was to understand light-absorption of complex entities as small and certainly not purely electrovalent entities.

There was nobody in 1951 who found it possible that crystal-field-theory, which had been known for many years at that time, would lead to an understanding of at least primarily covalent complexes.

It is quite typical that Van Vleck, who had the greatest merits in crystalfield-theory, together with Finkelstein in 1940 treated the chromium doublet and did not overcome the borderline (Ref. 19). These authors apparently could not see that the whole long-wave-length-region of the corresponding spectra could be explained by transitions between splitting products of the perturbed terms of the central ions. Nobody else had come so near to the border of ligand-field-theory at that time.

We in Frankfurt did not know the Van-Vleck-Finkelstein paper when we began our theoretical investigations.

Our ligand-field-theory certainly was not crystal-field-theory. So I do not understand why people prefer to call ligand-field-theory crystal-fieldtheory. At least I think there is no crystal or crystal-field in a complex. On the other hand the term ligand-field-theory today by many people is used for a molecular orbital theory of complexes where the valence electrons of the ligands are treated explicitly together with the electrons of the central ion. But in that case there is certainly no ligand field.

I don't like confusions and so I retain my terminology. I beg you to excuse that. 
3 .

Now I want to say a few words about the results of the ligand-field-theory.

At first let me point out that our theory is a characteristic model-theory and that means that constituent parts of the theoretical system like for example the ligand field have to be chosen carefully in accordance with the totality of all respective experiences. Model quantities like the ligandfield don't exist in nature. So they cannot be determined by experiments.

For example in the first paper one can read, that to get a suitable "ansatz" for the ligand-field in the case of the ion $\left[\mathrm{Ti}\left(\mathrm{H}_{2} \mathrm{O}\right)_{6}\right]^{3+}$ we have chosen for the water molecules a higher dipole moment than for the free water molecules. For arguments with an electrostatic basis, we thought one should include some polarisation effects.

From the statement made above one has to draw the conclusion that only results with a small sensitivity against changes of the model-parameters are really important. So for example the numbers and the sequence of the splitting-products of terms of the free ion for small and for high ligandfield intensities is rather independent of the specific form of the ligandfield-function. The number of the splitting-products is determined by the symmetry of the complex alone. For their sequence the sign of energyintegrals or in more complicated cases the sequence of their amounts also is responsible.

I shall pick out two typical examples to show you which types of results of ligand-field-theory in my opinion are and have always been the most important ones, namely propositions with yes-no-character in first line and propositions about pure numbers in second line.

\begin{tabular}{|c|c|c|c|c|c|c|c|c|c|c|c|}
\hline \multicolumn{2}{|c|}{$d^{\prime}$} & Th. & $\mathrm{Ti}^{3+}$ & \multicolumn{2}{|c|}{$d^{2}$} & Th. & $v^{3+}$ & \multicolumn{2}{|c|}{$d^{3}$} & Th. & $\mathrm{Cr}^{3+}$ \\
\hline \multicolumn{2}{|c|}{2} & -1 & + & \multicolumn{2}{|l|}{3} & $\begin{array}{l}+ \\
-\end{array}$ & $\begin{array}{l}+ \\
-\end{array}$ & \multicolumn{2}{|c|}{4} & $\begin{array}{l}+ \\
-\end{array}$ & $\begin{array}{l}+ \\
-\end{array}$ \\
\hline 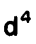 & Th. & $\mathrm{Mn}^{3+}$ & $\mathrm{Cr}^{2+}$ & $d^{5}$ & Th. & $\mathrm{Fe}^{3+}$ & $\mathrm{Mn}^{2+}$ & $d^{6}$ & Th. & $\mathrm{Co}^{3+}$ & $+\mathrm{Fe}^{2+}$ \\
\hline $\begin{array}{l}5 \\
3\end{array}$ & $\begin{array}{l}+ \\
+ \\
-\end{array}$ & $\begin{array}{l}+ \\
+ \\
-\end{array}$ & $\begin{array}{l}+ \\
+ \\
- \\
\end{array}$ & \begin{tabular}{l|}
6 \\
4 \\
2
\end{tabular} & $\begin{array}{l}+ \\
- \\
+\end{array}$ & $\begin{array}{l}+ \\
+ \\
\end{array}$ & $\begin{array}{l}+ \\
+ \\
+\end{array}$ & $\begin{array}{l}5 \\
3 \\
1\end{array}$ & $\begin{array}{l}+ \\
+\end{array}$ & $\begin{array}{l}+ \\
+\end{array}$ & $\frac{+}{+}$ \\
\hline 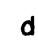 & & Th. & $\mathrm{Co}^{2+}$ & $d^{8}$ & & Th. & $\mathrm{Ni}^{2+}$ & $d^{s}$ & & Th. & $\mathrm{Cu}^{2+}$ \\
\hline$c$ & $z$ & $\begin{array}{l}+ \\
+\end{array}$ & $\begin{array}{l}+ \\
+\end{array}$ & 3 & & $\begin{array}{l}+ \\
-\end{array}$ & $\begin{array}{l}+ \\
-\end{array}$ & 2 & & + & + \\
\hline
\end{tabular}

Tab. 1

Groundstate multiplicities of octahedral complex ions

In each field of Table 1 (belonging to an electron configuration

a first column contains the multiplicities, which can be deduced in principle for $n$ d-electrons by spin alignment. The respective second column contains + and - signs as results of ligand-field-calculations of possible ground state multiplicities in the case of octahedral symmentry. $+(-)$ means that the respective multiplicity can (can not) really occur as real multiplicity of the ground state in an octahedral $d^{n}$-complex. The following column belongs to $d^{n}$-ions for which the multiplicities of the ground states of their octahedral complexes are known. The sign $+(-)$ means, that an octahedral complex with the respective groundstate multiplicity is (is not) known. You see a perfect agreement in a case where the theory has led to pure yes-nopredictions. 
A second example I pick out of the field of absorption-spectroscopy of transition metal complexes. Octahedral complexes of $\operatorname{Cr}(3+)$ besides of a very weak intercombination show two bands in the visible and the near ultraviolet region. I want to speak about the variations in the reach of the one of those two bands which lies at longer wave lengths, if one goes over from $\left[\mathrm{Cr} \mathrm{A}_{6}\right]{ }^{3+}$ to $\left[\mathrm{Cr} \mathrm{A}_{5} \mathrm{~B}\right]{ }^{2+}$, $\operatorname{cis}\left[\mathrm{Cr} \mathrm{A}_{4} \mathrm{~B}_{2}\right]+$ and trans $\left[\mathrm{Cr} \mathrm{A}_{4} \mathrm{~B}_{2}\right]^{+}$ The theoretically determined term systems (Ref. 21) (Fig.1) should be valid if $A$ is roughly $a$ dipole and $B$ an ion. For the case of the monoacido compound $\left[\mathrm{Cr} \mathrm{A}_{5} \mathrm{~B}\right]{ }^{2+}$ the term system contains the prediction, that the original band should be split into a band lying at the place of the original band and a second band shifted to longer wavelengths. This prediction is essentially of yes-no-character. In contrast to that behaviour for the case of cis [ $\left.\mathrm{Cr} \mathrm{A}_{4} \mathrm{~B}_{2}\right]+$ at the place of the original band now there should lie no band. That again is a yes-no-prediction.

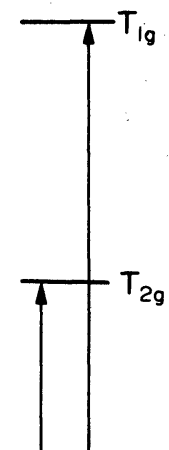

I II

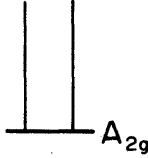

$\mathrm{O}_{\mathrm{h}}$

$\left[\mathrm{CrA}_{6}\right]^{3+}$

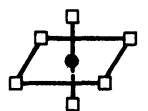

Fig. 1

Lower parts of term systems of $\mathrm{Cr}(3+)$-ions in

fields of different symmetry

The comparison with the observed spectra (Ref. 22) Fig. 2 and Fig. 3 show that both yes-no-predictions are right.

The splitting of the original octahedral band in the case of trans $\left[\mathrm{Cr} \mathrm{A}_{4} \mathrm{~B}_{2}\right]+$ should be of the same type as in the case of the monoacido compound trans [ $\left.\mathrm{Cr} \mathrm{A}_{4} \mathrm{~B}_{2}\right]^{+}$. This is a yes-no-prediction in agreement with the observed spectrum. The splitting width in the case of trans [Cr $\left.\mathrm{A}_{4} \mathrm{~B}_{2}\right]+$ is predicted twice as great as in the case [ $\left.\mathrm{Cr} \mathrm{A}_{5} \mathrm{~B}\right]{ }^{2+}$. This statement which is in rather good agreement with the observations belongs to the class of predictions, where the results of the theory are characterized by pure numbers.

With these few examples I wanted to point out which types of results according to my opinion were most important also for the further development of theoretical complex chemistry. Nevertheless overestimation of quantitative aspects and so called "successful" treatment of small effects could be observed in the literature. 

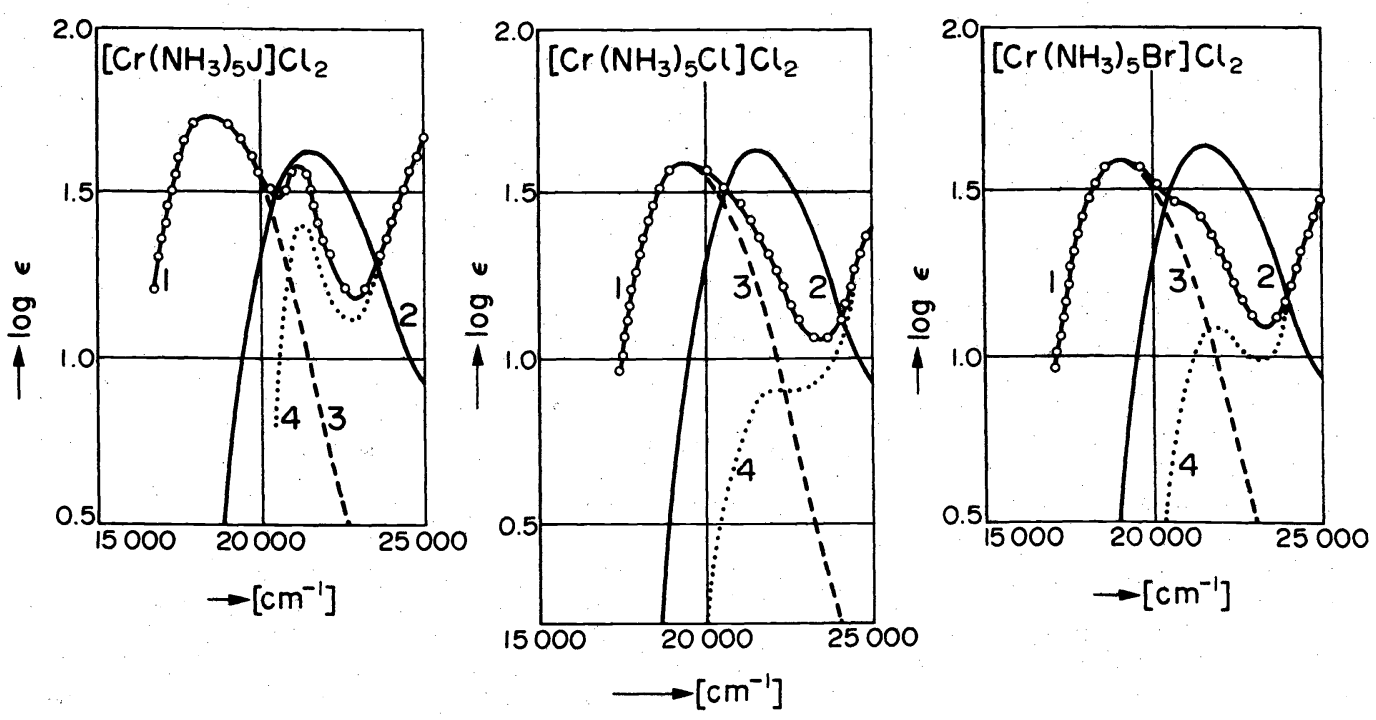

Fig. 2

Light absorption of $\mathrm{Cr}(3+)$ pentamino-acido-complexes in the region of the octahedral long-wavelength-band.

curve 1 = halogerio complex (expt 1 .); curve $2=\left[\mathrm{Cr}\left(\mathrm{NH}_{3}\right)_{6}\right]^{3+}$; curve 3 = result of a Gaussian analysis;

curve 4 = difference between curves 1 and 3 .

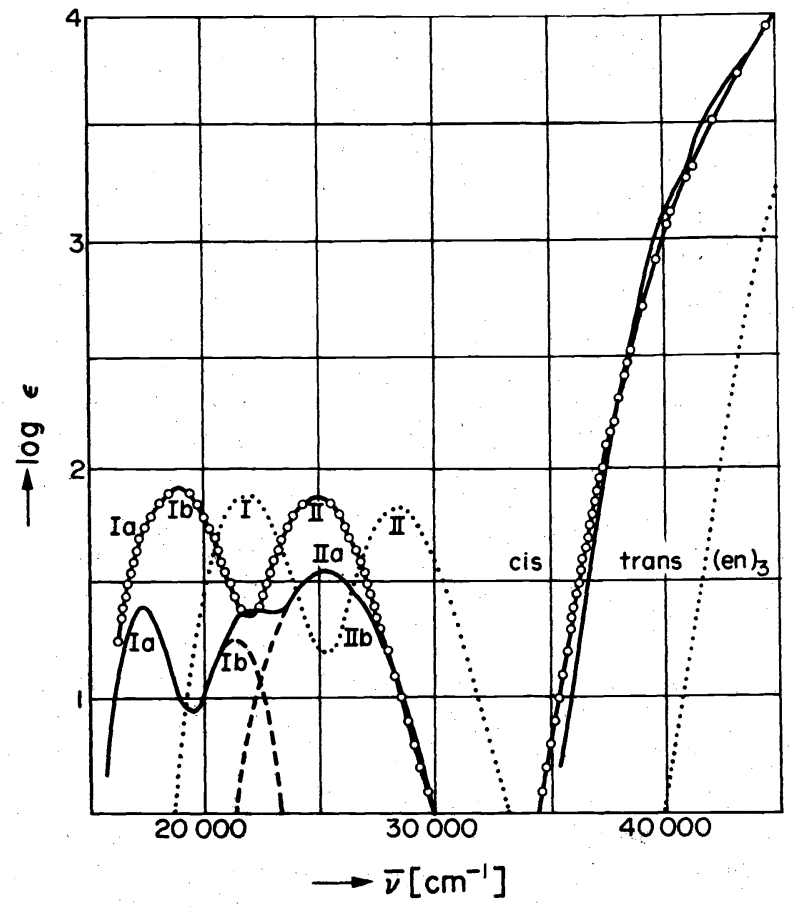

Fig. ${ }^{3}$ abst absorption of cis- and trans- $\left[\mathrm{Cr} \text { en } \mathrm{Cl}_{2}\right]^{+}$.

The light absorption of $\left[\mathrm{Cr} \mathrm{en}_{3}\right]^{3+}$ is added for reference. 
K.F. Gauss once remarked that too sharp reckoning is a sign for defective mathematical education. I think an analogous statement could be proved for the overestimation of quantitative aspects in science.

But ligand-field-theory in its development had to suffer also from misunderstanding of the underlying quantum mechanics.

According to the general procedure of ligand-field-theory for the case of a strong field Tanabe and Sugano (Ref. 23) for octahedral ions and FischerWasels (Ref. 24) for the historically interesting case of tetragonal $\mathrm{Ni}$-complexes have studied the ground states of complexes quite correctly.

Afterwards for the case of strong ligand-fields a so called "strong-fieldmethod" has been recommended by some people. They assumed that this procedure would be more suited in the case of a strong ligand-field, whereas our method from now on was called "weak-field-method".

In reality as long as equal one electron atomic orbitals are used, the application of the "strong-field-method" even in the case of a strong ligandfield leads to results which are generally worse than those obtained with the complete weak-field-method for the same case. However, some complex chemists apparently even now have not reached a real understanding of the problem.

$$
4 .
$$

After having recalled typical traits of ligand-field-theory by focussing attention on a few examples of its development I want to say that the word "ligand-field-theory" marks a certain step in the evolution of theoretical complex chemistry. Apparently it has been a necessary step and I think that there is an analogy between ligand-field-theory and Hückel's Mo-theory of $\pi$-electron systems, in the sense that later developments scarcely would have been possible without these essential steps.

At the end of the article written by Moffit and Ballhausen (Ref. 25) for Review of Physical Chemistry you can find a statement about the value of theories of that kind for chemistry.

As I said at the beginning a description of the following period would consume a lot of time. So only a few concepts, ideas and names may lead you to subsequent activities in theoretical complex chemistry.

Certainly, for example, it has been important to study Jahn-Teller-consequences in the domain of ligand-field-theory. A very happy idea about nephelauxetic phenomena has been introduced by Schäffer (Ref. 26) to whom also later investigations of angular overlap have to be attributed.

From the point of view of systematic quantum chemistry the next step was the reintroduction of valence electrons of the ligands and that means the development of the MO-theory of complex entities. So in some cases the relation of experimentally determined quantities to suitably chosen parameters has been reached also in cases, which could not be sufficiently described within the ligand-field-theory.

$\mathrm{Ab}$ initio calculations have been performed. Certainly they are not useless - and that inspite of the fact that the question of convergence in all cases is still open. Practical parametrization is helpful for practical chemistry.

Ligand-field-theory has been proved to be useful for the chemist. But what can theoreticians learn from the story? I want to discuss the answer to this question with a diagram. (Fig. 4).

The full circle may indicate symbolically the so called physical reality. The open circle describes a special model theory. The theoretician can choose different model theories at the outset, which in the symbolical diagram can be represented by open circles at different positions in the symbolic plane. From each model theory one can reach the physical reality step after step in the sense of higher approximations. 


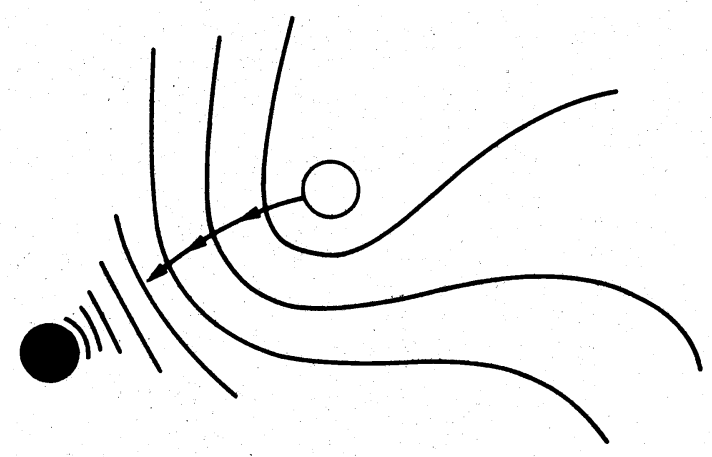

Fig. 4

Physical reality and model theories

If the open circle is close to the physical reality the model theory from the outset leads to a good knowledge in so far as calculations on that basis give rather good values for quantities like energy and so on. On the other hand in the symbolic plane there exists a field of complexity (inverse to simplicity) which we have marked in the diagram by lines of equal complexity. If the open circle lies at a point of high simplicity, that means, that the chosen model theory allows a good understanding of the essential phenomena. Now the experiences in theoretical complex chemistry show again that in the average a complementarity principle between the "knowledge"- and the "understanding"-quality of a theory is valid.

Normally it is impossible to have a theory which enables the theoretician to understand quite clearly all essential interrelations and to make good quantitative calculations at the same time.

Nevertheless the theoretician may have a good chance because our complementarity principle is valid only in the average. So it may be, that a theory can be found, which is rather close to the physical reality and nevertheless is astonishingly simple.

Such a rare case seems to be ligand-field-theory.

5.

Now, as announced, some personal facts.

In 1930 when I was sixteen years old I got as a Christmas gift from my parents a small booklet written by Professor Louis Dede in Giessen. The title was "Komplexchemie" and the essential point for me was that it contained a section on the Magnus theory from 1922. That exerted a great influence on me. In $1933 \mathrm{I}$ became a student of chemistry and my first teachers were Prof. Manchot and Prof. Hieber, both famous complex chemists. In Munich I became extremely fascinated by Prof. Sommerfeld and Quantum Theory. The result was a publication in 1940, authors Geheimrat Sommerfeld and $I$, a very unequal pair of men. Another consequence: ever since 1934 I lived with the book "Gruppentheorie und Quantenmechanik" written by Hermann Weyl. So it was natural that I came into contact with the group theoretical paper by Hans Bethe which had been published when he worked with Sommerfeld around 1930. In Munich it became very fruitful for me that I found the book "Chemische Bindung als elektrostatische Erscheinung" written by van Arkel and de Boer and translated into German by Wilhelm and $\mathrm{Li} \mathrm{Klemm}$ in 1931. Klemm's book on magneto chemistry contained also some electrostatic ideas.

Yet in Frankfurt in 1942 I met Prof. Gleu, an inorganic chemist, who had a thorough knowledge of the theory of group representations. At the same time began my friendship with Prof. Madelung, which lasted till his death in 1972. You remember that Madelung was one of the fathers of electrostatic ideas in chemistry. During the war-time I received the thesis written by Prof. Jannik Bjerrum about stability constants of complexes, 
which contained some nice spectra too, and I studied papers of Linhard about experimental complex spectroscopy. At the end of the war I lived in a small place in Oberhessen. There I met Prof. Albrecht Bethe, the father of Hans Bethe. With Albrecht Bethe, a physiologist, I had an excellent scientific contact. Later I met Hans Bethe, who visited his father.

After the end of the war the Frankfurt University was closed for a year. In that time G. Fritz, now professor of inorganic chemistry in Karlsruhe, my first doctorand, and F.E. Ilse came to my living place Laubach. I remember that we three together cut trees in the wood because otherwise it was impossible to escape the cold. Though we were rather hungry at that time we discussed vividly problems of theoretical complex chemistry and naturally also some other things.

In 1946 the university in Frankfurt was reopened and I found there as the new director of the institute of physical chemistry Prof. A. Magnus. He came from Leipzig. Prof. Bonhoeffer had taken him there because he had not been persona grata with the Nazis. In Frankfurt later after my coming back from Bonhoeffer's Max Planck-Institut für Physikalische Chemie, where I had worked as head of a department for $11 / 2$ years, I became the follower of Magnus. I have collected here some facts and I leave it to you to draw the lines to ligand-field-theory. And doing that, you should not forget, that the cooperation with Fritz Ilse has been essential.

It would easily be possible to add more names. I confine myself to mentioning as further essential cooperators Hansen, who died some years ago as a Professor of the Bonn-university, and Gliemann, who is now Professor in Regensburg. Among my friends from abroad I want to mention especially my Italian colleagues Sartori and Furlani, who very early acknowledged the meaning of ligand-field-theory.

All my cooperators from the physical chemistry institute in Frankfurt, who worked with me in the creative period, died or live at other places now. I finished my work in the field of complex chemistry in the early sixties and since that time. I have been active in other fields.

Finally some words about my personal relation to ligand-field-theory. Propositions in natural science contain concepts as elements. We cannot find concepts in nature. We have to invent them. But we are not free in doing that. Again and again we have to compare our inventions with our general experience to find out whether they are useful and which of them we should retain as elements for building up a science. So a natural science finally is a systematic description of natural phenomena with manmade concepts as elements.

Essentially an analogous statement holds for model theories. It is the scientist who chooses the open circle point in the symbolic plane of the last diagram - and not nature. The model is an invention of the scientist's spirit. The model cannot be found in nature because it does not exist in nature.

The early contact with Platon's ideas in my mind has finally led to the philosophical opinion about the structure of sciences and scientific reasoning which I have just described. So I can formulate my personal relation to ligand-field-theory in the following way:

I am happy that with my work in the field of theoretical complex chemistry I could contribute to chemistry a useful theoretical system, and it is very fortunate that all my experiences with complexes were in complete coincidence with my philosophical opinion about science.

\section{References:}

1. H. Hartmann, F.E. Ilse, Z. physik. Chem. 197, 239 (1951); Z. Naturforsch. 6a, 751 (1951).

2. A. Magnus, Z. anorg. allg. Chem. 124, 289 (1922); Physik. Z. 23, 241 (1922).

3. F.E. Ilse, Dissertation Univ. Frankfurt, "Quantenmechanische Rechnungen über Absorptionspektren polar aufgebauter anorganischer Komplexe", 1946. 
4. H.L. Schläfer, Dissertation Univ. Frankfurt, "Lichtabsorption wässriger Lösungen von Ti(3+)-Komplexverbindungen" 1950; H. Hartmann und H.L. Schläfer, z. Naturforsch. 6a, 760 (1951).

5. A. Werner, Neuere Anschauungen auf dem Gebiete der anorganischen Chemie, Braunschweig, (1905).

6. A. Kossel, Ann. d. Physik 49, 229 (1916).

7. R. Weinland, Einführung in die Chemie der Komplexverbindungen (Wernersche Koordinationslehre) in elementarer Darstellung, Stuttgart (1919).

8. G.N. Lewis, Journ. Am. Chem. Soc. 38, 762 (1916).

s.a.: G.N. Lewis, Die Valenz und der Bau der Atome und Moleküle, Braunschweig (1927).

9. I. Langmuir, Journ. Am. Chem. Soc. 38, 2221 (1916).

10. N.V. Sidgwick, Trans. Faraday Soc. 19, 472 (1923).

11. W. Heitler und F. London, Zs. f. Physik 44, 455 (1927).

12. L. Pauling, Journ. Am. Chem. Sos. 53, 1367 (1931).

13. F. Hund, Zs. f. Physik, 73, 1, 563 (1931).

R. Mulliken, Phys. Rev. 40, 55 (1932).

G. Herzberg, Leipziger Vorträge, (1931).

14. J.M. van Vleck, Journ. of Chem. Physics 1, 219 (1933).

15. R. Mulliken, Phys. Rev. 43, 279 (1933).

16. J.M. van Vleck and A. Sherman, Rev. mod. Physics, 7, 167 (1935).

17. E. Wigner, Zs. f. Physik, 40, 492 (1926).

18. H. Bethe, Ann. d. Physik 3, 133 (1929).

19. R. Finkelstein an J.M. van Vleck, J. Chem. Physics 8, 790 (1940).

20. H. Hartmann, Zs. f. Naturforsch. 11a, 884 (1956).

21. H. Hartmann und H.H. Kruse, Z. physik. Chem. (Neue Folge) 5,9 (1955).

22. M. Linhard und M. Weigel, Zs. physik. Chem. (Neue Folge) 5, 20 (1955).

23. Y. Tanabe und S. Sugano, J. phys. Soc. (Japan) 9, 763, 766 (1954).

24. H. Hartmann und H. Fischer-Wasels, Zs. phys. Chem. (Neue Folge)

$\underline{4}, 20$ (1955).

25. W. Moffitt und C.J. Ballhausen, Ann. Rev. physic. Chem. 7, 107 (1956).

26. C.E. Schäffer, ICCC Symposium, Rom (1957). 